\title{
La science régionale en perspective
}

\author{
Antoine Bailly \\ Université de Genève \\ RSAI Founder's Medal 2008
}

\section{Résumé}

Depuis sa création en 1954 la science régionale a d'abord contribué, sur le plan scientifique, à la reconstruction régionale de l'après guerre et à l'aménagement des territoires. Mais suite au libéralisme ambiant, elle a connu une grave crise dans les années 1990 avec la fermeture de nombreux départements dans le monde. Au $3^{\mathrm{e}}$ millénaire, avec une science régionale plus adaptée à l'évolution des sociétés et plus appliquée, elle retrouve une place essentielle dans la gestion des territoires. Cet article présente les grands axes de ce potentiel nouveau.

(c) 2009 Lavoisier, Paris. Tous droits réservés.

\section{Summary}

Perspectives in regional science. Since its foundation in 1954 regional science was first used for the reconstruction of regional economies after world war 2 and for regional planning. But, in the 1990's, with political liberalism, it entered into a deep crisis with the closing of many departments in the world. With the new millenium, a more applied regional science, taking into account the evolution of societies, find a new place in the scientific world and the planning of territories. This paper gives the main axis of this renewal

(C) 2009 Lavoisier, Paris. Tous droits réservés.

Mots clés : Science régionale, contexte politique et social, renouveau, mutations.

Keywords: Regional science, political and social contexts, renewal, mutations.

\footnotetext{
*Adresse email : antoine.bailly@unige.ch

doi:10.3166/ges.11.1-7 @ 2009 Lavoisier, Paris. Tous droits réservés.
} 
L'Association Internationale de Science Régionale a 55 ans en 2009, preuve de la capacité de cette nouvelle discipline à se renouveler. Dans sa préface de l'édition du cinquantième anniversaire (Golden Anniversary) des Papers in Regional Science (2003) Peter Batey parle d'un « stimulating and worthwhile field of intellectuel activity »... « constantly seeking new challenges ». C'est dans cette double direction que s'organise cet article, en retraçant les fondements de la science régionale et en analysant son potentiel après une période de crise à la fin du $\mathrm{XX}^{\mathrm{e}}$ siècle.

\section{Un retour dans le proche passé}

Si l'Association de Science Régionale a pu être créée en 1954, c'est bien grâce à un immense chercheur, Walter Isard, qui a mis en place (avec Alvin Hansen qu'on oublie trop souvent) en 1958, un Département de Science Régionale à l'Université de Pennsylvanie, où l'on va retrouver de grands noms : Thomas Reiner, Ben Stevens, Ron Miller, Julian Wolpert, chacun apportant une contribution originale au champ scientifique. Le premier doctorat, en 1960, fut celui de William Alonso, qui enseignera plus tard à Harvard et Berkeley.

Bien d'autres chercheurs contribuent à ce développement. Lloyd Rodwin, professeur de planification urbaine et régionale au M.I.T.à Boston, fervent défenseur de l'interdisciplinarité, François Perroux à Paris qui développe la théorie des pôles de croissance (1961), Claude Ponsard à Dijon qui rédige la première véritable histoire des théories spatiales (1958), replaçant les Johann von Thünen, Alfred Weber, Walter Christaller et August Lösch, dans le nouveau contexte disciplinaire, Wassily Leontieff qui développe les analyses matricielles "d'input - output », Jean Paelinck qui donne ses lettres de noblesse à l'économétrie spatiale (1983)... On pourrait citer bien d'autres noms, héritiers des pionniers allemands et créateurs de cette nouvelle discipline.

Il faut ajouter que la science régionale répondait clairement, dans les années 50 et 60 , à un besoin de société (Fig. 1) : contribuer, sur le plan scientifique, à la reconstruction économique de l'après-guerre; une vision soutenue par le gouvernement américain, qui voit dans la science régionale une discipline susceptible de réfléchir aux localisations stratégiques des entreprises et des ressources dans un contexte de " guerre froide ». Une vision aussi soutenue en Europe et en U.R.S.S. pour envisager un aménagement du territoire sur des bases scientifiques. La science régionale ainsi financée pouvait se développer rapidement grâce à ses associations nationales, en éclosion au début des années 1960. Ainsi avec J. Boudeville et F. Perroux, W. Isard peut-il lancer, en 1961, l'Association de Science Régionale de Langue Française dans des pays où la reconstruction passe par une réflexion sur le développement régional et le rôle des pôles de croissance (Derycke, 2001). Même si la discipline est dominée par l'abstraction et la modélisation, héritées de l'économie, les travaux sont variés et souvent très appliqués aux problèmes de développement et de convergence régionaux, et aux études de localisations d'activités économiques. On peut citer, entre autres, les travaux sur la localisation des activités sidérurgiques ou les missions de développement régional comme en Gaspésie au Québec (Office de Planification et de Développement du Québec).

De ce fait, cinq revues internationales de science régionale, en anglais, voient le jour les Papers in Regional Science, les Annals of Regional Science, l'International Regional Science Review, le Journal of Regional Science et Regional Science and 
Figure 1 :

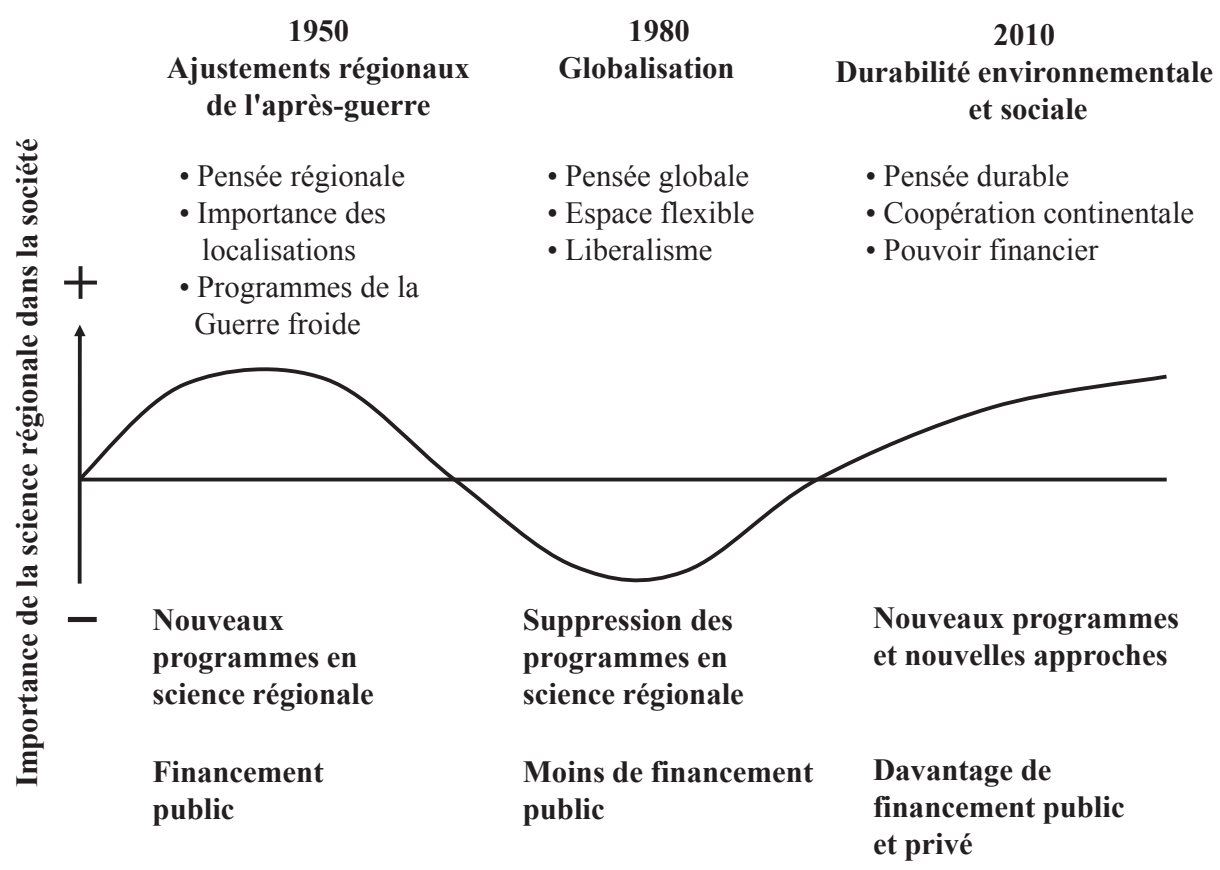

Urban Economics. Il faut y ajouter les revues dans d'autres langues comme la Revue d'Economie Régionale et Urbaine. Des collections d'ouvrages sont publiés chez les grands éditeurs Springer, Elsevier, Blackwell, Economica.... autour de trois grands thèmes : les théories de la localisation, les méthodes d'analyse régionale et spatiale, les politiques de développement régional.

Les ouvrages fondamentaux de Walter Isard Location and Space Economy (1956), Methods of Regional Analysis (1966), tout comme celui de William Alonso, Location and Land Use (1965), favorisent un enseignement structuré autour de méthodes précises, multiplicateurs économiques, programmation linéaire, calculs matriciels... D'autres publications en France, comme celles de François Perroux sur L'économie du XXe siècle, développent (1961) les modèles de gravité pour lancer le concept de pôle de croissance qui sera souvent employé pour les nouvelles localisations industrielles et les politiques de métropoles d'équilibre..

Beaucoup de chercheurs proches de la science régionale obtiennent des Nobels d'économie de Wassily Leontieff (1973) à Daniel McFadden (2000), moins connu, mais qui a fait évoluer les méthodes statistiques pour étudier les demandes de déplacements résidentiels et migratoires, en passant par Lawrence Klein (1980) pour le développement de modèles économétriques et leur application aux politiques économiques et Richard Stone (1984) pour le développement des systèmes de comptabilités nationaux. Ce n'est donc pas une surprise que de voir, en 2008, Paul Krugman, promoteur entre autre de la nouvelle géographie économique, recevoir un prix Nobel ! 


\section{Une période de crise}

En 1997, l'Empereur et l'Impératrice du Japon reçoivent les participants au Congrès Pacifique de Science Régionale, un véritable couronnement pour la science régionale... mais à cette époque la science régionale connaît une crise grave car le contexte politique a bien changé avec le développement des politiques économiques libérales dans la plupart des pays occidentaux (Fig. 1). La science régionale ne répond plus aux attentes d'une société ouverte au libéralisme, ce qui entraîne, dans un contexte de vive concurrence académique, la mise en cause et la fermeture de départements : S.P.U.R. à Louvain-la-Neuve, Département de Science Régionale de l'Université de Pennsylvanie en 1994... qui fut pourtant le berceau de la discipline. Et le label C.N.R.S. est parfois contesté à des instituts de recherche régionale en France, suite aux querelles académiques et disciplinaires... L'ironie de l'histoire fait qu'au moment où le concept de région redevient pertinent, où se créent en Espagne, en Belgique des régions plus autonomes, où de nouveaux régionalismes naissent sur les cendres d'empires décadents dans l'ex-U.R.S.S.et en Yougoslavie, où des politiques de régionalisation et de décentralisation sont mises en place dans de nombreux pays occidentaux, la discipline semble toucher à sa fin.

Les jeunes régionalistes sont-ils prêts à répondre aux questions majeures que se posent les autorités politiques et à prendre la relève de la science régionale des années 50 et 60 ? Comment revivifier les économies des régions périphériques et assurer une convergence dans les pays et au sein des unions continentales ? Peut-on utiliser le savoir-faire acquis par la science régionale pour le transformer en actions d'aménagement du territoire dans le contexte libéral ? Reposant sur les modèles empruntés à l'économie et à la géographie quantitative, la science régionale a su développer modèles et théories pour comprendre la répartition de flux économiques au sein des régions. Elle a créé un arsenal méthodologique, particulièrement adapté à ces questions. Mais la prééminence de la théorie est devenue parfois un handicap lorsque les sociétés se posent comme questions celles de l'emploi, de la marginalisation, de la qualité de vie, de la qualité de l'environnement et du développement durable, des questions auxquelles certaines sciences humaines et naturelles sont mieux préparées que la science régionale traditionnelle. R. Stough, dans sa réponse à notre article «Regional science in crisis » (Bailly et Coffey, 1994), écrit qu' « il est étrange de constater qu'au moment où les régions deviennent si importantes, les bases théoriques et pratiques de la discipline sont engluées dans un champ épistémologique étroit ». Même s'il n'est pas de notre souhait de réamorcer le débat ancien sur la « scientificité » des sciences humaines, sur les limites entre «science et non-science », nous constatons que, à la fin du $\mathrm{XX}^{\mathrm{e}}$ siècle, le dogmatisme néo-positiviste dominant a constitué un handicap pour le renouvellement de la science régionale. Il va pourtant pouvoir être surmonté par le renouveau des problématiques et des méthodes.

\section{La science régionale au début du troisième millénaire}

Ce renouveau émerge là où l'on ne l'attendait pas. Non aux États-Unis où le nombre de « régionalistes » stagne, non dans les pays du nord de l'Europe malgré le rôle innovateur de Torsten Hägerstrand (What about people inregional science?, 1970), mais en Europe du Sud, au Canada, en Asie et en Amérique Latine... Des membres de la Regional Science 
Association International, profitant des nombreux congrès mondiaux et continentaux (World Congress de Lugano, 2000, de Port Elizabeth, 2004, de Sao Paulo, 2008) (Congrès bi-annuels et annuels de la Pacific Regional Science Association et de la European Science Association), développent une discipline plus adaptée à l'évolution sociale et plus appliquée. Délaissant la théorie pure, ils fondent un puissant courant de réflexion-conseil aux régions, aux municipalités et parfois aux entreprises. On peut leur reprocher un éloignement de la tradition walrasienne, mais non une absence de réalisme ni de prise de conscience du caractère appliqué de la discipline. À la D.A.T.A.R en France, avec Jean-Louis Guigou, à l'Institut National de la Recherche Scientifique Urbanisation au Québec, les travaux sur l'aménagement du territoire et la prospective spatiale se multiplient. Mais dans les pays fondateurs de la science régionale théorique subsiste un problème relevé par Lay Gibson (1994) : lorsque les systèmes universitaires tiendront mieux compte des réalités de la recherche appliquée... celle-ci aura un poids égale à celui de la recherche théorique.

Pour l'avenir de la science régionale, suivons à nouveau l'avis de Lay Gibson (1994) qui nous conseille de compléter la lecture des manuels récents comme celui de Mario Polese et Richard Shearmur, "Economie Urbaine et Régionale » (2005) par celui de Walter Isard, «Methods of Regional Analysis » (1960) pour constater que les liens entre théorie et pratique étaient étroits. Le fossé entre la science régionale académique et le monde des décideurs était loin d'être aussi large que maintenant. Quatre articles anciens mais fondamentaux, nous le rappellent. Tout d'abord la fameuse conférence présidentielle de Torsten Hägerstrand (1973), « Regional science is about people, not just about location », nous montre que la discipline doit contribuer à rendre le monde plus agréable à vivre. Stan Czamanski (1976) suit cette voie en indiquant que nous devons mieux nous attacher aux motivations individuelles et sociales, par exemple pour mieux protéger l'environnement. Morgan Thomas (1977), de ce fait, propose de faire de la science régionale une science de « space society » plutôt que de la « space economy»; et en 1992 R. Bolton ajoute que c'est à la prospérité des hommes, plutôt qu'à la prospérité des régions, qu'il faut s'attacher. Nos travaux sur les représentations spatiales et la géographie du bien-être (1981) vont aussi dans cette direction

Pour cet avenir, ouvrons-nous ensuite aux nouvelles demandes de la société. Peter Nijkamp, David Boyce et Daniel Shefer proposaient d'ailleurs, dès 1990, cinq thèmes majeurs à aborder :

- le vieillissement des sociétés

- la dégradation de l'environnement

- l'innovation technologique

- les nouvelles cartes du monde et les nouvelles identités

- les nouvelles infrastructures

On peut y ajouter les questions d'exclusion, de migrations, de qualité de vie, de convergence, de métropolisation, bien des thèmes régulièrement présentés maintenant dans les congrès de science régionale, tout comme ceux plus méthodologiques traitant des imbrications spatiales et temporelles.

Ajoutons encore la contribution majeure de l'ouvrage de Masahisa Fujita, Paul Krugman et Anthony Venables, «The Spatial Economy » que les auteurs considèrent dans la ligne du projet de Walter Isard. La nouvelle géographie économique parle des agglomérations comme le résultat de tensions entre les forces centripètes qui favorisent le concentration des activités économiques et les forces centrifuges qui poussent à la 
dispersion. En associant modèles théoriques et simulations informatiques pour traiter des propriétés dynamiques non linéaires, la nouvelle géographie économique fait le lien entre l'économie urbaine, régionale et commerciale, une voie importante pour la science régionale, bien suivie par Jean-Marie Huriot et Jacques Thisse.

Parmi les autres voies, citons celle ouverte par l'ouvrage de Michael Porter, "The Competitiveness Advantage of Nations », reposant sur le concept de «cluster » et la compétitivité des entreprises. La concentration des entreprises gagnantes, stimulées par le milieu local serait une des causes de leur succès. On retrouve ici la théorie des milieux développée par Denis Maillat et Roberto Camagni (2006). On le constate, le potentiel est là, multiple, à la fois théorique et appliqué, utilisé dans des programmes comme celui de l'I.N.R.A. en France intitulé Pour et Sur le Développement Régional.

\section{En guise de conclusion}

Les voies pour l'avenir de la science régionale sont nombreuses comme l'illustre la Figure 2, avec ses prises en compte de l'économie, de la société et de l'environnement. Outre celles esquissées dans cet article, il faut constater que le nouveau contexte politique et social favorise à nouveau la discipline. Une prospective pour un monde des régions est demandé par des institutions mondiales, nationales et régionales. Celle-ci traite des inégalités et de la difficulté de la convergence économique et sociale, des conséquences des migrations et du vieillissement, de la métropolisation, de l'impact des infrastructures de transport, de l'innovation et de la gouvernance sur la santé et la qualité de vie ... Autant de thèmes qu'il faudra aborder avec la rigueur voulue par Walter Isard, l'humanité de Torsten Hagerstrand et la vision globale de Jean Paelinck.

Figure 1 :

\section{Le triangle de la science régionale}

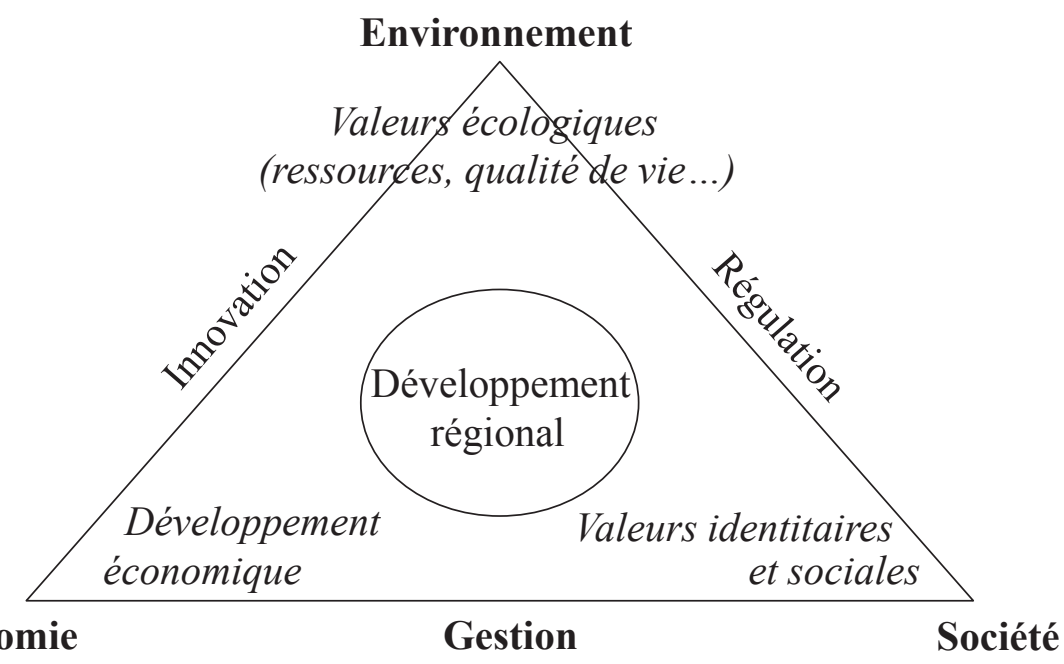




\section{Références}

ALONSO W. 1961, Location and Land Use, Harvard University Press, Cambridge.

BAILLY A.S., 1981, La géographie du bien-être, PUF, Paris

BAILLY A.S., GIBSON L.A., HAYNES K., 2008, Applied Geography fort the Entrepreneurial University, Economica, Paris.

BAILLY A.S., COFFEY W.J., 1994, «Regional Science in Crisis : A Plea for a more Open and Relevant approach», Papers in Regional Science, 73, 1, pp. 3-14.

BAILLY A.S., COFFEY W.J., GIBSON L.J., 1996, «Regional science back to the future», The Annals of Regional Science, 30, pp. 153-163.

CAMAGNI R., MAILLAT D., eds., 2006, Milieux innovateurs. Théories et politiques, Economica, Paris.

CUADRADO J.R., 1997, « Presidential Address», Papers in Regional Science, 76, 3, I-vii

DERYCKE P.H., 2001, Quarante ans de science régionale francophone, A.S.R.D L.F., Paris.

FUNK R., 1991, «Regional Science in transitio», Papers in Regional Science, 70, pp. 1-8.

GIBSON L, 1994, «Fixing the Fix we are», Papers in Regional Science, 79, 1, pp. 19-25.

GIBSON L., 1998, «Institutionalizing Regional Science», Presidential Address, Western RSA, Monterey, Cal.

HAGERSTRAND T., 1973, «What about people in Regional Science ?» Papers of the RSA, 24, pp. 7-21.

HURIOT J.M., 1994, Von Thünen. Economie et espace, Economica, Paris.

HURIOT J.M., THISSE J.F., 2000, The Economics of Cities : Theroretical Perspectives, Cambridge, Cambridge University Press.

ISSERMAN A.M., 1993, «Lost in Space ? On the History, Status and Future of Regional Science», Review of Regional studies», 23, pp. 1-50.

ISARD W., 1956, Location and Space Economy, .Cambridge , M.I.T. Press.

ISARD W., 1972, Méthodes d'analyse régionale. Paris, Dunod, D'abord paru en anglais en 1960, sous le tire : Methods of Regional Analysis, Cambrige ,M.I.T. Press.

ISARD W., 1975, Introduction to Regional Science. Englewood Cliffs, Prentice-Hall.

ISSERMAN A.M., 1995, «The History, Status and Future of Regional Science. An American Perspective», International Regional Science Review, 17, pp. 249-296.

JENSEN R.C., 1991, «Quo vadis, Regional Science ?», Papers in Regional Science, 70, pp. 97-111.

KRUGMAN P., 1995, Development, Geography, and Economic Theory, Cambridge , M.I.T. Press.

KUZNETS S., 1965, Towards a Theory of Economic Growth. New York, Norton.

LACOUR C., PUISSANT S., 1999, La métropolisation. Paris, Anthropos.

PAELINCK J.H.P., SALLEZ A., eds. 1983, Espace et localisation, Economica, Paris.

PERROUX F., 1961, L'économie du XXème siècle, P.U.F., Paris.

PLANE D.A., 1994, “On Discipline and Disciplines in Regional Science”, Papers in Regional Science, 73, 1, pp. 19-23.

POLESE M., SHEARMUR R., 2005, Economie urbaine et régionale, Economica, Paris.

PONSARD C., 1958, Histoire des théories économiques spatiales, Colin, Paris.

RODWIN L., 1987, «On the Education of Urban and Regional Specialists : A comparative perspective», Papers of the RSA, 62, pp. 1-11.

SCHAFFER W.A., 1997, «Regionalists, Regional Science, And Love», Papers in Regional Science, 76, 2, pp. I-vii. 
